\title{
More on modal aspects of default logic*
}

\author{
V. Wiktor Marek ${ }^{\dagger}$ and Miroslaw Truszczynski ${ }^{\dagger}$ \\ Department of Computer Science \\ University of Kentucky \\ Lexington, KY 40506-0027 \\ marek@ms.uky.edu, mirek@ms.uky.edu
}

\begin{abstract}
Investigations of default logic have been so far mostly concerned with the notion of an extension of a default theory. It turns out, however, that default logic is much richer. Namely, there are other natural classes of objects that might be associated with default reasoning. We study two such classes of objects with emphasis on their relations with modal nonmonotonic formalisms.

First, we introduce the concept of a weak extension and study its properties. It has long been suspected that there are close connections between default and autoepistemic logics. The notion of weak extension allows us to precisely describe the relationship between these two formalisms. In particular, we show that default logic with weak extensions is essentially equivalent to autoepistemic logic, that is, nonmonotonic logic KD45.

In the paper we also study the notion of a set of formulas closed under a default theory. These objects are shown to correspond to stable theories and to modal logic S5. In particular, we show that skeptical reasoning with sets closed under default theories is closely related with provability in S5.

As an application of our results we determine the complexity of reasoning with weak extensions and sets closed under default theories.
\end{abstract}

\section{Introduction}

Default logic is an extension of classical propositional logic tailored to handle situations where information is incomplete and the agent is arriving at conclusions using both presence and absence of information. In this paper we study two classes of objects that can be associated with a default theory: weak extensions and closed theories. We will investigate connections of these classes with expansions in modal nonmonotonic logics and with models of logic programs.

Let us assume that an agent is to reason about a certain domain $\mathcal{D}$. It is commonly accepted that an inference engine of an agent should include propositional calculus in

${ }^{*}$ Parts of this paper appeared earlier in the extended abstract Relating Autoepistemic and Default Logics, published in the Proceedings of the First International Conference on Principles of Knowledge Representation and Reasoning, KR89, Morgan-Kaufmann, 1989.

$\dagger$ This work was partially supported by by National Science Foundation under grant IRI-9012902, National Science Foundation and the Commonwealth of Kentucky EPSCoR program under grant RII 8610671 
the language generated by the set of propositional variables relevant to the domain $\mathcal{D}$. We will denote this language by $\mathcal{L}$. In addition, an agent's reasoning mechanism should contain some domain-specific rules describing what is known about the domain $\mathcal{D}$. These rules are of the form

$$
\frac{\varphi_{1}, \ldots, \varphi_{n}}{\vartheta}
$$

where $\varphi_{1}, \ldots, \varphi_{n}, \vartheta$ are formulas of the underlying language $\mathcal{L}$. A rule of the form $(1)$ works as follows: once all the premises $\varphi_{1}, \ldots, \varphi_{n}$ of the rules are derived, the agent concludes $\vartheta$. Due to assumed omniscience of agents, theories we will consider in this paper are closed under propositional provability. Thus, without loss of generality, we will restrict to rules with only one premise, that is, to rules of the form

$$
\frac{\varphi}{\vartheta}
$$

The difference between a rule of the form (2) and the implication

$$
\varphi \supset \vartheta
$$

is that although both permit the derivation of $\vartheta$ out of $\varphi$, the rule of the form (1) has no side-effects. For instance, the formula $\neg \vartheta \supset \neg \varphi$ cannot be derived from the rule (1). On the other hand, it can certainly be derived from the implication $\varphi \supset \vartheta$. Speaking informally, using rules rather than implications allows us to control the flow of information more tightly.

Let $D$ be a collection of rules of the form (2). A large part of the theory of propositional logic as well as the theory of formal systems can be lifted to the proof system which allows to derive formulas using the rules from $D$ along with propositional calculus. We will denote such a proof system by $P C+D$. The system $P C+D$ can be formally specified by the following definition of the notion of a proof or derivation. A proof of a formula $\varphi$ from $W$ in the system $P C+D$ is a finite sequence of formulas $\varphi_{1}, \ldots, \varphi_{n}$ such that $\varphi_{n}=\varphi$ and for every $i, 1 \leq i \leq n$,

$\mathbf{P 1} \varphi_{i}$ is a tautology, or $\varphi_{i} \in W$

P2 $\varphi_{i}$ follows by modus ponens from some formulas $\varphi_{i_{1}}, \varphi_{i_{2}}, i_{1}, i_{2}<i$ or

P3 there is a rule

$$
\frac{\varphi}{\varphi_{i}}
$$

in $D$, such that $\varphi=\varphi_{j}$ for some $j<i$.

By $C n^{D}(W)$ we will denote the set of all formulas possessing a proof from $W$ in $P C+D$.

While the notion of a proof yields a "bottom-up" description of $C n^{D}(W)$, there is another, "top-down", characterization. A theory $U \subseteq \mathcal{L}$ is closed under a rule $\frac{\varphi}{\vartheta}$ if $\vartheta \in U$ whenever $\varphi \in U$. Similarly, a theory $U$ is closed under modus ponens if for every formula $\psi$ and $\varphi, \psi \in U$ whenever $\varphi \in U$ and $\varphi \supset \psi \in U$. A theory $U$ is closed under the system $P C+D$ if

1. $U$ is closed under the rules from $D$,

2. $U$ is closed under modus ponens, 
3. $U$ contains all tautologies.

By taking the intersection of all theories containing a theory $W \subseteq \mathcal{L}$ and closed under the system $P C+D$, we obtain the least theory with these properties. It is easy to see that this least theory containing $W$ and closed under $P C+D$ is exactly equal to $C n^{D}(W)$. Hence, the closure approach yields an equivalent "top-down" description of the set $C n^{D}(W)$. We will see later in the paper that once we extend the concept of a rule to capture incomplete information, the equivalence of the "bottom-up" and "top-down" approaches will no longer hold.

Default logic can be viewed as a generalization of the concepts described above. Specifically, by a default we mean an object of the form

$$
\frac{\alpha: M \beta_{1}, \ldots, M \beta_{k}}{\vartheta}
$$

where $\alpha, \beta_{1}, \ldots, \beta_{n}, \vartheta \in \mathcal{L}$. A most commonly accepted interpretation of a rule of the form (3) is: "If $\alpha$ has been established, and $\beta_{1}, \ldots, \beta_{k}$ are consistent, then derive $\vartheta$ ".

This is the approach taken by Reiter in his important paper [Rei80]. But in order to obtain the Reiter's approach, one has to clarify two concepts appearing in our informal understanding of the meaning of a default. One needs to say what is meant by "established" and the word "consistent" must get a precise meaning. A trick to obtain Reiter's default logic is to select a theory $S \subseteq \mathcal{L}$. This theory $S$ is used to control consistency. That is, "consistency" is interpreted as "consistency with $S$ ". Next, to make precise the meaning of the word "established", the notion of an $S$ proof is introduced. Namely, given a set of defaults $D$, a formula $\varphi \in \mathcal{L}$ and a set of formula $W \subseteq \mathcal{L}$, a sequence $\varphi_{1}, \ldots, \varphi_{n}$ is an $S$-proof of $\varphi$ from $W$ by means of defaults in $D$ if $\varphi$ if $\varphi_{n}=\varphi$ and for every $i \leq n$ :

DP1 $\varphi_{i}$ is a tautology, or $\varphi_{i} \in W$ or

DP2 $\varphi_{i}$ follows by modus ponens from some formulas $\varphi_{i_{1}}, \varphi_{i_{2}}, i_{1}, i_{2}<i$ or

DP3 there is a rule

$$
\frac{\varphi_{i_{1}}: M \beta_{1}, \ldots, M \beta_{k}}{\varphi_{i}}
$$

with $i_{1}<i, \neg \beta_{1} \notin S, \ldots, \neg \beta_{k} \notin S$.

By $C n^{D, S}(W)$ we will denote the set of all formulas possessing an $S$-proof from $W$ by means of defaults in $D$. A formula is "established" if it has an $S$-proof.

A pair $(D, W)$, where $D$ is a set of defaults and $W$ is a subset of , is called a default theory. A theory $S \subseteq \mathcal{L}$ is called an extension (the notion was introduced by Reiter [Rei80]) of a default theory $(D, W)$ if $S$ is precisely the set of formulas possessing an $S$-proof from $W$ by means of defaults in $D$. That is, $S$ is an extension of $(D, W)$ if $S=C n^{D, S}(W)$.

Reiter's extensions turned out to have numerous applications. For example, they capture such concepts as stability in logic programming ([BF91, MT89]), abduction ([KKT92]) and truth maintenance ([RDB89, MT93]). But the enormous success of Reiter's approach had also the effect of narrowing the scope of investigations in default logic to extensions and their properties. Only recently, along with better understanding of nonmonotonicity of commonsense reasoning, new versions of default logic have been proposed: cumulative default logic by Brewka [Bre91] and default logic 
with well-founded semantics by Baral and Subrahmanian [BS91]. It is quite obvious that there will be many others to follow.

In this paper we will introduce and investigate two classes of objects that can be associated with default theories. Each yields an interesting version of the default logic. These classes arise as generalizations of "bottom-up" and "top-down" characterizations of the operator $C n^{D}$ of the system $P C+D$. We have already presented one generalization of the "bottom-up" approach. The notion of an $S$-proof generalizes the notion of a proof in a system $P C+D$ and was used as the basis for our definition of extensions. But it turns out that the notion of a proof can be generalized in more than one way. Another possibility is obtained by weakening the condition DP3. In this manner we get the concepts of weak S-proof and of weak extension.

We study the properties of these notions in our paper. We prove that an extension of $(D, W)$ is a weak extension of $(D, W)$, but not necessarily conversely. Thus, the structure of weak extensions captures Reiter's extensions but provides additional "points of view" that can be associated with a default theory. We investigate the relationship between weak extensions and expansions in modal nonmonotonic logics. We show that weak extensions are in a perfect correspondence with autoepistemic expansions. Hence, default logic with a slightly changed set of structures allows us to capture autoepistemic logic (or, equivalently, the nonmonotonic modal logic KD45).

Another class of objects that can be associated with default theories can be obtained by generalizing the notion of a closure under a rule, that is, by adopting the "top-down" approach. This leads us to the notion of sets of formulas closed under default theories. These objects are also investigated in the paper. As pointed above, for a set $D$ of monotone rules and a subset $W \subseteq \mathcal{L}$, there exists the least set of formulas containing $W$ and closed under $D$ and propositional provability. It is no longer the case when $D$ consists of defaults. It is easy to construct default theories with more than one minimal theory closed under a default theory $(D, W)$. In contrast with extensions and weak extensions sets of formulas closed under a default theory always exist. For example, the whole language $\mathcal{L}$ is closed under any default theory. Moreover, by an easy application of a Zorn lemma one can show that for every default theory there exist minimal sets of formulas closed under it. These minimal sets of formulas appeared for the first time in the paper by Hanks and McDermott [HM86]. The problem of existence of a consistent set of formulas closed under a default theory is less trivial. We show in this paper that it is equivalent to the problem of satisfiability in the modal logic $\mathbf{S 5}$.

In this paper we concentrate on the question of interpretability of the notions of weak extensions and sets closed under default theories within modal nonmonotonic systems. We show that default logic with weak extensions can be embedded into autoepistemic logic (that is, nonmonotonic logic KD45) and that the notion of a closed theory is related to a class of theories closed under modal logic S5. Rather surprisingly, our results have corresponding converse versions. Speaking informally, it turns out that autoepistemic logic can be expressed within default logic with weak extensions and modal-free $\mathbf{S} 5$-consequences of modal theories are expressible within default logic by means of closed theories.

We also briefly discuss relations between weak extensions and closed theories and objects studied in logic programming. We point out that, under a natural translation, weak extension define supported Herbrand models of the completion of the program, and closed theories correspond to Herbrand models of the completion. 


\section{Preliminaries from modal logic}

In this section we recall some basic facts on modal logic and its nonmonotonic versions. By the language of modal logic we mean an extension of the propositional language $\mathcal{L}$ by one modal operator $L$. We call this language $\mathcal{L}_{L}$. An intuitive interpretation of $L \varphi$ is $\varphi$ is known or $L \varphi$ is believed. A formula $\neg L \neg \varphi$ will often be abbreviated to $M \varphi$

A theory $T \subseteq \mathcal{L}_{L}$ is stable if it satisfies the following conditions:

St1 $T$ is closed under propositional provability;

St2 $\varphi \in T$ implies $L \varphi \in T$;

St3 $\varphi \notin T$ implies $\neg L \varphi \in T$.

A stable theory is uniquely determined by its subset consisting of modal-free (or objective) formulas, that is $T \cap \mathcal{L}$. For a theory $S \subseteq \mathcal{L}$, by $S T(S)$ we denote the stable theory $T$ such that $T \cap \mathcal{L}=C n(S)$.

Given a formula $\varphi \in \mathcal{L}_{L}$, we say that $\varphi$ is an autoepistemic clause (ae-clause for short) if $\varphi$ is of the form

$$
L \alpha \wedge M \beta_{1} \wedge \ldots \wedge M \beta_{m} \supset \gamma
$$

with $\gamma \in \mathcal{L}$. We allow a possibility that in an ae-clause of the form (4) the conjunct $L \varphi$ or all conjuncts of the form $M \beta_{i}$ are missing. In particular, every formula $\gamma \in \mathcal{L}$ is an ae-clause.

The restriction to just one conjunct of the form $L \alpha$ is not restrictive since a formula

$$
L\left(\alpha_{1} \wedge \ldots \wedge \alpha_{k}\right) \equiv\left(L \alpha_{1} \wedge \ldots \wedge L \alpha_{k}\right)
$$

is a theorem of every normal modal logic.

It is easy to see that for every theory $I \subseteq \mathcal{L}_{L}$ there exists a theory $I^{\prime}$ consisting of ae-clauses such that $C n(I)=C n\left(I^{\prime}\right)$.

We say that an ae-clause is simple if $\alpha, \beta_{1}, \ldots, \beta_{m} \in \mathcal{L}$.

We will deal with two normal modal logics in this paper: KD45, which is based on the schemata K, D, 4 and 5, and S5 based on the schemata K, T, 4 and 5. Since the axiom schema D holds in S5, S5 is an extension of KD45. We refer the reader to the monograph by Hughes and Cresswell [HC84] for more details.

Following Moore ([Moo85]), we call a theory $T \subseteq \mathcal{L}_{L}$ an autoepistemic or stable expansion of a theory $I$ if

$$
T=C n(I \cup\{L \varphi: \varphi \in T\} \cup\{\neg L \psi: \psi \notin T\})
$$

It is easy to see that a stable expansion of any theory must itself be a stable theory (that is must satisfy conditions ( $\mathbf{S t 1})-(\mathbf{S t 3})$ ). Moreover, if $T=S T(S)$ then $T$ is a stable expansion of $S$.

Given a modal logic $\mathcal{S}$, and a theory $I \subseteq \mathcal{L}_{L}$, a theory $T \subseteq \mathcal{L}_{L}$ is called an $\mathcal{S}$-expansion of $I$ if

$$
T=C n_{\mathcal{S}}(I \cup\{\neg L \psi: \psi \notin T\})
$$

Here, by $C n_{\mathcal{S}}$ we mean the consequence operator permitting application of the necessitation rule of proof to all formulas, not only the axioms of $\mathcal{S}$. 
As long as $I$ is $\mathcal{S}$-consistent (that is $\left.C n_{\mathcal{S}}(I) \neq \mathcal{L}_{L}\right), \mathcal{S}$-expansions of $I$ are stable and consistent.

The solutions to the equation (5), that is stable expansions "almost" coincide with KD45-expansions. Speaking formally, we have the following result.

Proposition 2.1 (Schwarz, [Shv90]) Consistent autoepistemic expansions coincide with KD45-expansions. That is, if $I \subseteq \mathcal{L}_{L}$, then a consistent theory $T$ is a solution to equation (5) if and only if $T$ is a solution to equation (6) with $\mathcal{S}=\mathbf{K D} 45$.

Logic KD45 permits to reduce in a formula the degree of nesting of the operator $L$ to at most 1 . This property implies the following "normal form" result.

Proposition 2.2 (Konolige, [Kon88]) For every theory $I \subseteq \mathcal{L}$ there is a theory $I^{\prime}$ consisting of simple ae-clauses such that $C n_{\mathbf{K D 4 5}}(I)=C n_{\mathbf{K D} 45}\left(I^{\prime}\right)$. Consequently, $I$ and $I^{\prime}$ have precisely the same consistent autoepistemic expansions, and they are included in the same stable theories.

Let $I \subseteq \mathcal{L}_{L}$ be a set of ae-clauses. Let $T \subseteq \mathcal{L}_{L}$ be a stable theory. We say that the ae-clause

$$
L \alpha \wedge M \beta_{1} \wedge \ldots \wedge M \beta_{m} \supset \gamma
$$

from $I$ is a generating formula for $T$ if

$$
\alpha \in T \text { and } \neg \beta_{1}, \ldots, \neg \beta_{m} \notin T
$$

By $G F(I, T)$ we denote the set of all ae-clauses from $I$ that are generating for $T$. Finally, $c(G F(I, T))$ is the set of all consequents of the ae-clauses from $G F(I, T)$. Notice that by the definition of ae-clauses $c(G F(I, T)) \subseteq \mathcal{L}$.

We have the following description of autoepistemic expansions of theories consisting of ae-clauses ([MT91]).

Proposition 2.3 Let $I \subseteq \mathcal{L}_{L}$ be a theory consisting of ae-clauses and let $T$ be a stable theory. Then $T$ is a stable expansion of $I$ if and only if

$$
T=S T(c(G F(I, T)))
$$

Stable theories can be characterized as solutions of the equation (6) with $\mathcal{S}=\mathbf{S 5}$. Specifically, we have the following result.

Proposition 2.4 (McDermott [McD82]) A theory $T$ is stable and consistent if and only if $T=C n_{\mathbf{S 5}}(\{\neg L \psi: \psi \notin T\})$. That is, if and only if $T$ is an $\mathbf{S 5 - e x p a n s i o n}$ of $\emptyset$. For a theory I which is $\mathbf{S 5}$-consistent, consistent $\mathbf{S 5 - e x p a n s i o n s ~ o f ~ I ~ a r e ~ p r e c i s e l y ~}$ stable and consistent theories containing $I$.

With every modal logic $\mathcal{S}$ we assign a nonmonotonic version of $\mathcal{S}$ (nonmonotonic $\mathcal{S}$ for short) as follows. We define the nonmonotonic $\mathcal{S}$-consequences (or skeptical consequences) of $I$ as

$$
C n_{\mathcal{S}}^{n}(I)=\bigcap\{T: T \text { is an } \mathcal{S} \text {-expansion of } I\}
$$

In the case of $\mathcal{S}=\mathbf{S} 5$, Proposition 2.4 implies that $C n_{\mathcal{S}}^{n}(I)=C n_{\mathcal{S}}(I)$ for every $I \subseteq \mathcal{L}_{L}$ 
The skeptical mode of reasoning can be introduced in a much more general setting. Suppose that we have an operator $E$ which assigns to theories (modal, default, propositional, etc.) a family of theories (expansions, extensions, weak extensions, models). Then, the skeptical reasoning consequence operator with respect to the mapping E can be defined by

$$
C O N S^{E}(I)=\bigcap\{T: T \in E(I)\} .
$$

In the paper we will discuss properties of skeptical reasoning with respect to weak extensions and sets closed under default theories.

As mentioned in the introduction, in this paper we study the relationship between versions of default logic and modal nonmonotonic logics. To this end we need a way of translating default theories into theories in the modal language $\mathcal{L}_{L}$. One such interpretation was proposed by Konolige [Kon88]. According to his approach, a default

$$
d=\frac{\alpha: M \beta_{1}, \ldots, M \beta_{k}}{\gamma}
$$

is assigned a simple ae-clause

$$
e m b(d)=L \alpha \wedge M \beta_{1} \wedge \ldots \wedge M \beta_{k} \supset \gamma .
$$

and a default theory $(D, W)$ is assigned a modal theory

$$
\operatorname{emb}(D, W)=W \cup\{e m b(d): d \in e m b(d)\} .
$$

Other interpretations are also possible, for instance we can assign to the same default the formula

$$
L \alpha \wedge L M \beta_{1} \wedge \ldots \wedge L M \beta_{k} \supset \gamma .
$$

This second approach was introduced and studied in [Tru91]. In particular, it was shown there that by means of such interpretation default logic with extensions can faithfully be represented within the nonmonotonic logic $\mathbf{S 4 F}$.

In this paper, we focus on the translation proposed by Konolige. In addition to the interpretation $e m b$, we consider also the inverse operator $e m b^{-1}$. It is defined for an arbitrary simple ae-clause $\varphi$. If $\varphi \in \mathcal{L}, \operatorname{emb}^{-1}(\varphi)=\varphi$. If $L$ appears in a simple ae-clause $\varphi$, say $\varphi$ is of the form (4), then

$$
e m b^{-1}(\varphi)=\frac{\alpha: M \beta_{1}, \ldots, M \beta_{k}}{\gamma} .
$$

Finally, for a theory $I$ consisting of simple ae-clauses we define $e m b^{-1}(I)=(D, W)$, where $W=I \cap \mathcal{L}\}$ and $D=\left\{e m b^{-1}(\varphi): \varphi \in I \backslash \mathcal{L}\right\}$.

\section{Weak extensions}

In this section we will introduce another variant of a context-dependent proof. This time a context will be used not only to check consistency of the justifications of a default but also the validity of its prerequisite. The resulting notion of a weak $S$-proof will then be used as the basis for our definition of another class of objects, called weak extensions, that can be associated with default theories. 
Definition 3.1 Let $W$ and $S$ be subsets of $\mathcal{L}, D$ a set of defaults and $\varphi$ a formula in $\mathcal{L}$. By a weak $S$-proof of $\varphi$ from $W$ by means of defaults in $D$ (or, simply a weak $S$-proof of $\varphi$, if $W$ and $D$ are understood) we mean a sequence $\varphi_{1}, \ldots, \varphi_{n}$ of formulas from $\mathcal{L}$ such that $\varphi=\varphi_{n}$ and for every $i, 1 \leq i \leq n$,

WDP1 $\varphi_{i}$ is a tautology,

WDP2 $\varphi_{i}$ follows by modus ponens from formulas $\varphi_{j_{1}}$ and $\varphi_{j_{2}}$, for some $j_{1}, j_{2}<i$,

WDP3 there is a default

$$
\frac{\alpha: M \beta_{1}, \ldots, M \beta_{m}}{\varphi_{i}}
$$

in $D$ such that $\alpha \in S$ and, for every $j, 1 \leq j \leq m, \neg \beta_{j} \notin S$.

The set of all formulas possessing a weak $S$-proof from a theory $W$ by means of defaults in $D$ will be denoted by $C n_{\text {weak }}^{D, S}(W)$. It has a nice description in terms of the propositional consequence operator $\mathrm{Cn}$. Consider a default

$$
\frac{\alpha: M \beta_{1}, \ldots, M \beta_{m}}{\gamma} .
$$

Let us call it $d$. The default $d$ is called generating with respect to a context $S$ if

1. $\alpha \in S$, and

2. $\neg \beta_{i} \notin S$, for all $i, 1 \leq i \leq m$.

By $G D(D, S)$ we will denote the subset of $D$ consisting of defaults generating with respect to $S$. It easily follows by induction on the length of proof that a formula $\varphi$ has a weak $S$-proof from $W$ by means of defaults in $D$ if and only if

$$
\varphi \in C n(W \cup c(G D(D, S))) .
$$

In other words, the set of all formulas having a weak $S$-proof from $W$ by means of defaults in $D, C n_{\text {weak }}^{D, S}(W)$, satisfies

$$
C n_{\text {weak }}^{D, S}(W)=C n(W \cup c(G D(D, S))) .
$$

As we have seen the notion of an $S$-proof gives rise to the concept of extension. In a similar way another class of objects can be defined by means of weak $S$-proofs.

Definition 3.2 Let $(D, W)$ be a default theory and let $S$ be a subset of $\mathcal{L}$. The theory $S$ is a weak extension of $(D, W)$ if $S$ is precisely the set of formulas possessing a weak $S$-proof from $W$ by means of defaults from $D$. That is, $S$ is a weak extension of $(D, W)$ if and only if

$$
S=C n(W \cup c(G D(D, S))) .
$$

Let us compare the definitions of $S$-proof and weak $S$-proof. Clearly, conditions DP1 and WDP1 as well as DP2 and WDP2 coincide. The only difference is in the third condition. In the case of weak $S$-proofs we no longer require that the prerequisite of a default be proven prior to the application of the default. It is enough that it belongs 
to the context $S$. This modification of the third condition introduces a possibility of "circular" proofs. For example, given a default

$$
\frac{p: M q}{p}
$$

where $p$ and $q$ are two different propositional variables, if an agent chooses to consider a context containing $p$ (chooses to have $p$ in its knowledge set), then it can provide an a posteriori justification for its choice by means of a weak $S$-proof of $p$. A very similar reasoning pattern is possible in autoepistemic logic, which suggests the existence of close connections between the notions of weak extension and autoepistemic expansion. Indeed, such connections can be found and we will discuss them in detail below.

Weak extensions behave, in general, nonmonotonically. In classical logic, when a formula is added to a theory, the class of models of the resulting theory is a subset of the class of models of the original one. It is not so for weak extensions of default theories. For example, consider a theory $(D, W)$, where $W=\emptyset$ and $D=\left\{\frac{: M p}{\perp}\right\}$. This theory has no weak extension. Let us define now $W^{\prime}=W \cup\{\neg p\}$. It is easy to see that the new default theory has one weak extension, $C n(\{\neg p\})$. In particular, it follows that the skeptical reasoning associated with weak extensions is nonmonotonic:

$$
C O N S^{w \cdot e x t}(D, W) \nsubseteq C O N S^{w \cdot e x t}\left(D, W^{\prime}\right)
$$

Let us also observe that weak extensions for a default theory, unlike extensions, do not in general form an antichain. It is easy to see that a default theory $(D, W)$, where $W=\emptyset$ and $\left.D=\frac{p: M q}{p}\right\}$ has two weak extensions $C n(\emptyset)$ and $C n(\{p\})$.

Next, we will show now that every weak extension is an extension. To this end, we will first investigate the relationship between $S$-proofs and weak $S$-proofs. The term weak $S$-proof may be somewhat misleading. In general, it is not the case that every $S$-proof is a weak $S$-proof. However, for some contexts $S$, in particular for those that are extensions, it is so.

Proposition 3.1 Let $W$ and $S$ be subsets of $\mathcal{L}$ and $D$ a set of defaults. If

(1) $S$ contains every formula that has an $S$-proof from $W$ by means of defaults in $D$

then every $S$-proof is a weak $S$-proof. In particular, the condition (1) is satisfied if $S$ is an extension of $(D, W)$.

Proof: In our argument by an $S$-proof (weak $S$-proof) we mean an $S$-proof (weak $S$-proof) from $W$ by means of defaults in $D$. Let us consider an $S$-proof $\varphi_{1}, \ldots, \varphi_{n}$. Assume that every $S$-proof of length less than $n$ is a weak $S$-proof. In particular, it follows that $\varphi_{1}, \ldots, \varphi_{n-1}$ is a weak $S$-proof. Hence, it is enough to show that at least one of the conditions WDP1 - WDP3 is satisfied for $\varphi_{n}$. If $\varphi_{n}$ is a tautology or belongs to $W$, the condition WDP1 holds. If $\varphi_{n}$ follows from some $\varphi_{i}, \varphi_{j}$, where $i, j<n$, by modus ponens, then the condition WDP2 holds. The last possibility is that there is a default

$$
\frac{\alpha: M \beta_{1}, \ldots, M \beta_{m}}{\varphi_{n}}
$$

such that $\alpha=\varphi_{j}$ for some $j<n$ and $\neg \beta_{k} \notin S$, for every $k, 1 \leq k \leq m$. Since $\alpha$ has an $S$-proof, by the condition (1) it follows that $\alpha \in S$. Consequently, the condition WDP3 is satisfied for $\varphi_{n}$. 
Suppose now that $S$ is an extension for $(D, W)$. Then, by the definition, $S$ is exactly the set of formulas possessing an $S$-proof from $W$ by means of defaults from $D$. Hence, (1) holds.

As a corollary, we obtain the property that every extension of a default theory is a weak extension of this default theory, too.

Corollary 3.2 Let $(D, W)$ be a default theory. For every theory $S \subseteq \mathcal{L}$, if $S$ is an extension for $(D, W)$ then $S$ is a weak extension of $(D, W)$.

Proof: Assume that $S$ is an extension of $(D, W)$. Then, every formula in $S$ has an $S$-proof from $W$ by means of defaults in $D$. By Proposition 3.1, every element in $S$ has a weak $S$-proof from $W$ by means of defaults in $D$. Hence, $S \subseteq C n_{\text {weak }}^{D, S}(W)$. On the other hand, one can easily show that since $S$ is an extension, $S$ contains the consequents of all defaults in $D$ that are generating with respect to $S$. In addition, $S$ contains $W$ and is closed under propositional provability. Hence $S \supseteq C n(W \cup$ $c(G D(D, S)))=C n_{\text {weak }}^{D, S}(W)$. Consequently, $S=C n_{\text {weak }}^{D, S}(W)$, that is, $S$ is a weak extension of $(D, W)$.

The converse statement is not true. Consider a default theory $\Delta=\left(\left\{\frac{p:}{p}\right\}, \emptyset\right)$. It is not hard to see that $\Delta$ has two weak extensions: $C n(\emptyset)$ and $C n(\{p\})$. Only the first of them is an extension for $\Delta$. The other one is not.

To conclude this section, let us note that if all the defaults in $D$ are prerequisitefree then, clearly, the notions of an $S$-proof and a weak $S$-proof by means of defaults from $D$ coincide. Consequently, we obtain the following result.

Theorem 3.3 Let $D$ be a set of prerequisite-free defaults. Then, for every theory $W \subseteq \mathcal{L}$, a theory $S \subseteq \mathcal{L}$ is an extension for $(D, W)$ if and only if $S$ is a weak extension for $(D, W)$.

\section{Weak extensions and autoepistemic logic}

The existence of close connections between default and autoepistemic logics has been suspected for a long time. Most of the effort has been focused on relating extensions and expansions (see, for example, [Kon88]). This has proved to be a difficult task and no simple modal interpretation of defaults is known under which there would be a one-to-one correspondence between these two concepts. The reason seems to be, speaking informally, that extensions do not allow "circular" justifications while expansions do. The results of this section show that our modification of the notion of an extension so that "circular" arguments become possible, is precisely what is needed in order to obtain a version of default logic equivalent with autoepistemic logic.

We will show that default logic with weak extensions can be represented as a fragment of autoepistemic logic concerned with theories without nested modalities. Quite surprisingly, the converse result is also true. We will show that default logic with weak extensions is powerful enough to represent full autoepistemic logic (with one, rather minor caveat to be discussed in detail below). In particular, no restrictions on the depth of nesting of the modality are needed. As the basis of our representability results we choose the modal interpretation of defaults by means of the formula (9). However, we would like to stress that our results remain valid for other natural modal interpretations of defaults, for example, for the interpretation given by (10). 
First, we show that weak extensions can be represented as autoepistemic expansions.

Theorem 4.1 Let $(D, W)$ be a default theory. Let $S \subseteq \mathcal{L}$ be closed under propositional consequence. The theory $S$ is a weak extension for $(D, W)$ if and only if $S T(S)$ is an autoepistemic expansion for $\operatorname{emb}(D, W)$.

Proof: It is easy to see that a default $d$ is generating with respect to $S$ if and only if the formula $\operatorname{emb}(d)$ is generating with respect to $S T(S)$. Indeed, say

$$
d=\frac{\alpha: M \beta_{1} \ldots, M \beta_{m}}{\gamma} .
$$

Assume that $d$ is generating with respect to $S$. Then, $\alpha \in S$ and $\beta_{i} \notin S$, for every $i, 1 \leq i \leq m$. Since $S$ is closed under propositional provability, $S=S T(S) \cap \mathcal{L}$. Hence, $\alpha \in S T(S)$ and $\beta_{i} \notin S T(S)$, for every $i, 1 \leq i \leq m$. Consequently, the formula $e m b(d)$ is generating with respect to $S T(S)$. The converse implication can be proved in a similar way.

In addition, every element of $W$ is a generating formula with respect to $S T(S)$. Hence, we have that

$$
W \cup c(G D(D, S))=G F(\operatorname{emb}(D, W)) .
$$

The assertion is now a direct consequence of (12), (13) and Theorem 2.3,

Theorem 4.1 has an immediate corollary about expressibility of autoepistemic logic in default logic, by means of weak extensions, if one restricts attention to theories consisting of simple ae-clauses.

Corollary 4.2 Let $I \subseteq \mathcal{L}_{L}$ consist of simple ae-clauses. A stable theory $T \subseteq \mathcal{L}_{L}$ is an autoepistemic expansion of $I$ if and only if $T \cap \mathcal{L}$ is a weak extension of emb $b^{-1}(I)$.

The proof of Proposition 2.2 (see [Kon88]) implies, in fact, that there is an algorithm that for every modal theory $I$ produces a modal theory $I^{\prime}$ consisting of simple ae-clauses with exactly the same consistent autoepistemic expansions as $I$. Combining this result with Corollary 4.2 implies an algorithm which assigns to every autoepistemic theory $I$ without inconsistent expansions a default theory $\Delta_{I}$ in such a way that autoepistemic expansions of $I$ correspond to weak extensions of $\Delta_{I}$.

Theorem 4.3 A consistent stable theory $T$ is an autoepistemic expansion of a modal theory $I$ if and only if $T \cap \mathcal{L}$ is a consistent weak extension of the default theory $\Delta_{I}=e m b^{-1}\left(I^{\prime}\right)$, where $I^{\prime}$ is as in Proposition 2.2.

This result says that full autoepistemic logic can be represented within default logic with weak extensions. There is only one minor restriction. The algorithm implied by the proof of Proposition 2.2 does not preserve inconsistent expansions. That is, the inconsistent expansion of a modal theory $I$ may no longer be an expansion of $I^{\prime}$. Consequently, under our translation $I \mapsto \Delta_{I}$, the inconsistent expansion of $I$ may not have a corresponding inconsistent weak extension of $\Delta_{I}$. In all other cases the correspondence is perfect.

The situation is even nicer in the case of skeptical mode of reasoning. Namely, it turns out that modal-free consequences of a theory $I$ under the skeptical autoepistemic mode of reasoning are exactly the consequences of the default theory $\Delta_{I}$ under the skeptical reasoning with weak extensions. 
Corollary 4.4 For every modal theory I

$\mathcal{L} \cap \bigcap\{T: T$ is an expansion of $I\}=\bigcap\left\{S: S\right.$ is a weak extension of $\left.\Delta_{I}\right\}$.

Proof: Notice that the intersection of all members of the empty family of theories equals $\mathcal{L}_{L}$. Hence,

$\bigcap\{T: T$ is an expansion of $I\}=\bigcap\{T: T$ is a consistent expansion of $I\}$.

Thus, the assertion follows from Theorem 4.3.

\section{Closure under defaults and logic S5}

In this paper we regard default logic as a generalization of formal proof systems $P C+$ $D$ containing propositional logic and some additional (monotone) rules of inference. Reasoning in such systems can equivalently be defined by means of the concept of a proof and by means of the notion of the closure under the rules of proof in $D$. The first approach can be generalized to the case of default logic in at least two ways. The notion of an $S$-proof leads to extensions and the notion of a weak $S$-proof yields weak extensions studied in the previous section. We will investigate now the second approach. A theory $S \subseteq \mathcal{L}$ is closed under a default

$$
\frac{\alpha: M \beta_{1}, \ldots, M \beta_{m}}{\gamma}
$$

if $\alpha \in S$ and $\neg \beta_{i} \notin S, 1 \leq i \leq m$, implies that $\gamma \in S$. A theory $S$ is closed under a default theory $(D, W)$ if

C1 $W \subseteq S$;

C2 $S$ is closed under propositional provability;

C3 $S$ is closed under every default in $D$.

First, we will show that every extension of a default theory $(D, W)$ is a minimal theory closed under $(D, W)$.

Theorem 5.1 Let $(D, W)$ be a default theory. I a theory $S \subseteq \mathcal{L}$ is an extension of $(D, W)$ then $S$ is a minimal theory closed under $(D, W)$.

Proof: Since $S$ is an extension of $(D, W), W \subseteq S$ and $S$ is closed under propositional provability. To verify the condition $\mathrm{C} 3$, consider a default

$$
d=\frac{\alpha: M \beta_{1}, \ldots, M \beta_{m}}{\gamma} .
$$

Assume that $\alpha \in S$ and $\neg \beta_{i} \notin S, 1 \leq i \leq m$. Since $S$ is an extension of $(D, W), \alpha$ has an $S$-proof from $W$ by means of defaults in $D$. Hence, $\gamma$ has an $S$-proof from $W$ by means of defaults in $D$. Thus, $\gamma \in S$.

To prove minimality, consider a theory $U$ closed under $(D, W)$ and assume that $U \subseteq S$. It is easy to prove by induction on the length of a proof that

$$
U \supseteq C n^{D, U}(W) .
$$


In addition, since $U \subseteq S$,

$$
C n^{D, U}(W) \supseteq C n^{D, S}(W) .
$$

Since $S$ is an extension, $U \supseteq S$ follows. Hence, $U=S$.

The converse of the statement of Theorem 5.1 is not true. Consider a default theory $(D, W)$, where $W=\emptyset$ and $D=\left\{\frac{: M q}{p}\right\}$. Clearly, $C n(\{\neg q\})$ is a minimal theory closed under $(D, W)$. On the other hand, $C n(\{\neg q\})$ is not an extension (not even a weak extension) of $(D, W)$.

The assertion of Theorem 5.1 fails if extensions are replaced by weak extensions. As we have seen weak extensions do not form an antichain, in general, while minimal closed theories obviously form one.

Our results in the remainder of this section relate sets of formulas which are closed under a default theory $(D, W)$ with stable theories containing $\operatorname{emb}(D, W)$. In particular, we will show that skeptical reasoning with closed theories is related to monotonic reasoning in modal logic S5

Theorem 5.2 Let $(D, W)$ be a default theory. A theory $S \subseteq \mathcal{L}$ is closed under $(D, W)$ if and only if $S$ is closed under propositional provability and $\operatorname{emb}(D, W) \subseteq$ $S T(S)$.

Proof: Suppose that $S$ is closed for $(D, W)$. Then, $S$ is closed under propositional provability and $W \subseteq S$. Let $d$ be a default from $D$, say

$$
d=\frac{\alpha: M \beta_{1} \ldots, M \beta_{m}}{\gamma}
$$

We need to show that

$$
e m b(d)=L \alpha \wedge M \beta_{1} \wedge \ldots \wedge M \beta_{m} \supset \gamma
$$

belongs to $S T(S)$.

First, assume that $\alpha \notin S$. Then, since $S$ is closed under propositional provability, $\neg L \alpha \in S T(S)$. Since $S T(S)$ is closed under propositional provability, emb $(d) \in$ $S T(S)$. Next, assume that $\neg \beta_{i} \in S$, for some $i, 1 \leq i \leq m$. Then, $L \neg \beta_{i} \in S T(S)$. As before, since $S T(S)$ is closed under propositional provability, emb $(d) \in S T(S)$. Finally, assume that $\alpha \in S$ and, for every $i, 1 \leq i \leq m, \neg \beta_{i} \notin S$. ince $S$ is closed under the defaults from $D$, it follows that $\gamma \in S$. Hence, $\gamma \in S T(S)$ and, consequently, $e m b(d) \in S T(S)$. Thus, $\operatorname{emb}(D, W) \subseteq S T(S)$.

The converse implication can be proved in a similar fashion.

Theorem 5.2 allows us to relate skeptical reasoning by means of theories closed for a default theory $(D, W)$ with monotonic consequence operator for the modal logic $\mathbf{S 5}$. It follows from Proposition 2.4 that the intersection of all stable theories containing a given modal theory $I$ coincides with the set of S5-consequences of $I$. Hence, Theorem 5.2 implies the following result.

Theorem 5.3 Let $(D, W)$ be a default theory. A formula $\varphi \in \mathcal{L}$ belongs to the intersection of all theories closed for $(D, W)$ if and only if $\varphi$ is an $\mathbf{S 5 - c o n s e q u e n c e ~ o f ~}$ $\operatorname{emb}(D, W)$. 
Let us denote by CONS the consequence operator for the skeptical reasoning with closed theories. That is, given a default theory $(D, W)$, we denote

$$
\operatorname{CONS}(D, W)=\bigcap\{S: S \text { is closed for }(D, W)\}
$$

It follows from Theorem 5.3 that CONS is monotonic both in $D$ and $W$. Since the definition of $C O N S$ does not change if the intersection of all closed theories is replaced by the intersection of minimal closed theories, it follows from our results that the skeptical mode of reasoning corresponding to the class of minimal closed theories of Hanks and McDermott is monotone.

As in the case of weak extensions we have the following two results on expressibility the concept of stability by means of closed theories.

Corollary 5.4 Let $I \subseteq \mathcal{L}_{L}$ consist of simple ae-clauses. A stable theory $T$ contains $I$ if and only if $T \cap \mathcal{L}$ is closed for $e m b^{-1}(I)$.

Theorem 5.5 Let $I \subseteq \mathcal{L}_{L}$ be an arbitrary modal theory. A stable theory $T$ contains $I$ if and only if $T \cap \mathcal{L}$ is closed for the default theory $\Delta_{I}=e m b^{-1}\left(I^{\prime}\right)$, where $I^{\prime}$ is as in Proposition 2.2.

Theorem 5.5 implies the converse result to Theorem 5.3. Namely, it shows that computing modal-free S5-consequences of an arbitrary modal theory $I$ can be performed in default logic by means of theories closed for a certain default theory.

Theorem 5.6 Let $I \subseteq \mathcal{L}_{L}$ be an arbitrary modal theory. A formula $\varphi \in \mathcal{L}$ is an S5-consequence of $I$ if and only if $\varphi$ is in the intersection of all theories closed for emb $b^{-1}\left(I^{\prime}\right)$, where $I^{\prime}$ is as in Proposition 2.2.

\section{Complexity issues}

In this section we will briefly discuss complexity issues related to weak extensions and closed theories. For all the undefined notions on complexity theory the reader is referred to the monograph by Garey and Johnson [GJ79] and to the papers by Gottlob [Got92] and Niemelä [Nie92], where the questions of complexity of problems involving extensions of default theories and autoepistemic expansions are studied.

First, we will deal with the case of weak extensions. We will consider the following five problems:

(PR1) given a finite default theory $(D, W)$, decide if $(D, W)$ has a weak extension. (PR2) given a finite default theory $(D, W)$ and a formula $\varphi$ decide if there is a weak extension of $(D, W)$ which contains $\varphi$.

(PR3) given a finite default theory $(D, W)$ and a formula $\varphi$ decide if there is a weak extension of $(D, W)$ which does not contain $\varphi$.

(PR4) given a finite default theory $(D, W)$ and a formula $\varphi$ decide whether $\varphi$ belongs to all weak extensions of $(D, W)$.

(PR5) given a finite default theory $(D, W)$ and a formula $\varphi$ decide whether $\varphi$ is in no extension of $(D, W)$.

It is evident that problems PR1 - PR3 are in $\Sigma_{2}^{P}$. For example, let us consider problem PR2. To prove that PR2 is in $\Sigma_{2}^{P}$ we need to show that there is a polynomialtime nondeterministic Turing machine with an oracle for a problem from the class NP 
which decides PR2. It is indeed the case. Such a machine would first (nondeterministically) guess a subset $D^{\prime}$ of defaults and then would check, using the propositional satisfiability oracle that

$$
G D\left(D, C n\left(W \cup\left\{c(d): d \in D^{\prime}\right\}\right)\right)=D^{\prime},
$$

that is, that $C n\left(W \cup\left\{c(d): d \in D^{\prime}\right\}\right)$ is a weak extension, and that

$$
\varphi \notin C n\left(W \cup\left\{c(d): d \in D^{\prime}\right\}\right) .
$$

Clearly, only polynomially many calls to the oracle are needed.

Since problems PR4 and PR5 are the complements of the problems PR3 and PR2, respectively, they both are in $\Pi_{2}^{P}$. Completeness of the problems PR1 - PR3 in $\Sigma_{2}^{P}$ and PR4 and PR5 in $\Pi_{2}^{P}$ follows from the corresponding results on extensions [Got92]. All default theories constructed in the proofs given by Gottlob in [Got92] contain only prerequisite-free defaults and for such theories the notions of extension and weak extension coincide (Theorem 3.3). Hence, we obtain the following result.

Theorem 6.1 Problems PR1 - PR3 are $\Sigma_{2}^{P}$-complete. Problems PR4 and PR5 are $\Pi_{2}^{P}$-complete.

Let us pass on to the case of closed theories. Here, the existence problem is trivial. Namely, for every default theory $(D, W)$ there exists a theory closed for $(D, W)$. If we ask for the existence of a consistent closed theory for $(D, W)$, by Theorem 5.3 , it is equivalent to $\mathbf{S 5}$-consistency of $\operatorname{emb}(D, W)$ an thus, it is NP-complete (the satisfiability problem for the logic $\mathbf{S 5}$ is NP-complete, see [Lad77]). Similarly, the problem whether a formula $\varphi \in \mathcal{L}$ is in all closed theories for $(D, W)$ is equivalent to the question whether $\varphi$ is an $\mathbf{S 5}$-consequence of $\operatorname{emb}(D, W)$, which is the complement of the $\mathbf{S 5}$-satisfiability problem and, hence, is co-NP-complete. Summarizing, we get the following theorem.

Theorem 6.2 Given a finite default theory $(D, W)$ it is NP-complete to decide if there is a consistent theory closed for $(D, W)$. Given a formula $\varphi \in \mathcal{L}$ and a finite default theory $(D, W)$ it is co-NP-complete to decide if $\varphi$ is in all theories closed for $(D, W)$.

\section{Models and supported models of logic programs}

In this section we will briefly discuss connections of weak extensions and closed theories with models of propositional logic programs.

By a (propositional) logic program we mean a set $P$ of program clauses, that is, expressions of the form

$$
C=p \leftarrow q_{1}, \ldots, q_{n}, \operatorname{not}\left(s_{1}\right), \ldots, \operatorname{not}\left(s_{m}\right)
$$

where $p, q_{i}$ and $s_{i}$ are atomic formulas of our propositional language $\mathcal{L}$.

There are several ways in which a clause of the form (14) can be interpreted as a default. Following [BF91, MT89] we assign to a clause of the form (14) the default

$$
d(C)=\frac{q_{1} \wedge \ldots \wedge q_{n}: M \neg s_{1}, \ldots, M \neg s_{m}}{p}
$$


We assign to a program $P$ a default theory

$$
d t(P)=(d(P), \emptyset)
$$

where $d(P)=\{d(C): C \in g(P)\}$. In [BF91, MT89] it has been proved that $M$ is a stable model of $P$ if and only if $C n(M)$ is a default extension of $d t(P)$. Weak extensions of $d t(P)$ have also a clear interpretation in this context. Recall that a supported model of a program $P$ is a set $M$ of atoms such that $M$ is a model of $P$ and, in addition, whenever an atom $p$ belongs to $M$ then there is a clause $C$ of the form (14) in $P$ such that $p$ is the head of $P$, and $M$ satisfies the body of $C$.

Not only we can interpret logic programs as default theories. We have seen that default theories can be interpreted as modal theories. Combining these embeddings yields a modal interpretation of logic programs. Namely, for a clause $C$ of the form (14) we define

$$
\operatorname{modal}(C)=L\left(q_{1} \wedge \ldots \wedge q_{n}\right) \wedge M \neg s_{1} \wedge \ldots \wedge M \neg s_{m} \supset p .
$$

For a program $P$, we define

$$
\operatorname{modal}(P)=\{\operatorname{modal}(C): C \in P\} .
$$

Reasoning as in the proof of Theorem 4.1 we obtain the following result.

Proposition 7.1 Let $M$ be a set of atoms. The following conditions are equivalent:

1. $M$ is a supported model of $P$.

2. $C n(M)$ is a weak extension of $d t(P)$.

3. $S T(M)$ is an autoepistemic expansion of modal $(P)$.

The equivalence of (1) and (3) is related to a result in [MS89], where modal characterization of supported models has been first investigated. The advantage of our approach over that of [MS89] is that the translation modal $(P)$ used here is modular (see [Imi87]) whereas the translation considered in [MS89] is not modular.

A similar result can be obtained for the class of all models of a program $P$ which turn out to correspond to closed theories and $\mathbf{S} 5$-expansions.

Proposition 7.2 Let $M$ be a set of atoms. The following conditions are equivalent:

1. $M$ is a model of $P$.

2. $C n(M)$ is closed under $d t(P)$.

3. $S T(M)$ contains modal $(P)$.

4. $S T(M)$ is a $\mathbf{S 5 - e x p a n s i o n ~ o f ~ m o d a l ~}(P)$. 


\section{References}

[BF91] N. Bidoit and C. Froidevaux. Negation by default and unstratifiable logic programs. Theoretical Computer Science, 78(1, (Part B)):85-112, 1991.

[Bre91] G. Brewka. Cumulative default logic: in defense of nonmonotonic inference rules. Artificial Intelligence, 50(2):183-205, 1991.

[BS91] C. Baral and V.S. Subrahmanian. Dualities between alternative semantics for logic programming and nonmonotonic reasoning (extended abstract). In A. Nerode, W. Marek, and V.S. Subrahmanian, editors, Logic programming and non-monotonic reasoning (Washington, DC, 1991), pages 69-86, Cambridge, MA, 1991. MIT Press.

[GJ79] M.R. Garey and D.S. Johnson. Computers and intractability. A guide to the theory of NP-completeness. W.H. Freeman and Co., San Francisco, Calif., 1979 .

[Got92] G. Gottlob. Complexity results for nonmonotonic logics. Journal of Logic and Computation, 2(3):397-425, 1992.

[HC84] G.E. Hughes and M.J. Cresswell. A companion to modal logic. Methuen and Co., Ltd., London, 1984.

[HM86] S. Hanks and D. McDermott. Default reasoning, nonmonotonic logics and frame problem. In Proceedings of AAAI-86, pages 328-333. Morgan Kaufmann, 1986.

[Imi87] T. Imieliński. Results on translating defaults to circumscription. Artificial Intelligence, 32(1):131-146, 1987.

[KKT92] A. C. Kakas, R. A. Kowalski, and F. Toni. Abductive logic programming. Journal of Logic and Computation, 2(6):719-770, 1992.

[Kon88] K. Konolige. On the relation between default and autoepistemic logic. Artificial Intelligence, 35(3):343-382, 1988.

[Lad77] R. E. Ladner. The computational complexity of provability in systems of modal propositional logic. SIAM Journal on Computing, 6(3):467-480, 1977.

[McD82] D. McDermott. Nonmonotonic logic II: nonmonotonic modal theories. Journal of the ACM, 29(1):33-57, 1982.

[Moo85] R.C. Moore. Semantical considerations on nonmonotonic logic. Artificial Intelligence, 25(1):75-94, 1985.

[MS89] W. Marek and V.S. Subrahmanian. The relationship between logic program semantics and nonmonotonic reasoning. In Logic programming (Lisbon, 1989), MIT Press Series in Logic Programming, pages 600-617, Cambridge, MA, 1989. MIT Press. 
[MT89] W. Marek and M. Truszczyński. Stable semantics for logic programs and default theories. In E.Lusk and R. Overbeek, editors, Proceedings of the North American Conference on Logic Programming, pages 243-256. MIT Press, 1989.

[MT91] W. Marek and M. Truszczyński. Autoepistemic logic. Journal of the ACM, 38(3):588-619, 1991.

[MT93] W. Marek and M. Truszczyński. Nonmonotonic Logic; Context-Dependent Reasoning. Springer, Berlin, 1993.

[Nie92] I. Niemelä. On the decidability and complexity of autoepistemic reasoning. Fundamenta Informaticae, 17(1-2):117-155, 1992.

[RDB89] M. Reinfrank, O. Dressler, and G. Brewka. On the relation between truth maintenance and non-monotonic logics. In Proceedings of IJCAI-89, pages 1206-1212, San Mateo, CA, 1989. Morgan Kaufmann.

[Rei80] R. Reiter. A logic for default reasoning. Artificial Intelligence, 13(1-2):81$132,1980$.

[Shv90] G.F. Shvarts. Autoepistemic modal logics. In R. Parikh, editor, Theoretical aspects of reasoning about knowledge (Pacific Grove, CA, 1990), Morgan Kaufmann Series in Representation and Reasoning, pages 97-109, San Mateo, CA, 1990. Morgan Kaufmann.

[Tru91] M. Truszczyński. Embedding default logic into modal nonmonotonic logics. In A. Nerode, W. Marek, and V.S. Subrahmanian, editors, Logic programming and nonmonotonic reasoning (Washington, DC, 1991), pages 151165, Cambridge, MA, 1991. MIT Press. 